\title{
Improving Outcomes for Adolescents With Drug-Resistant Tuberculosis
}

\author{
Lindsay McKenna ${ }^{1,}$; Erica Lessem ${ }^{1}$; Loyce Maturu ${ }^{2}$ \\ ${ }^{1}$ Treatment Action Group, TB/HIV Project, New York, USA \\ ${ }^{2}$ Africaid Zvandiri Program, Harare, Zimbabwe \\ *Corresponding author: Lindsay McKenna, Treatment Action Group, New York, USA. Tel: +1-2122537922, Fax: +1-2122537923, E-mail: Lindsay.McKenna@treatmentactiongroup.org
}

Received: May 29, 2015; Revised: May 31, 2015; Accepted: May 31, 2015

Keywords: Adolescent; Tuberculosis; Therapeutics

\section{Dear Editor,}

As Moyo and colleagues' research illustrated, we must improve outcomes for the estimated 655000 adolescents living with drug-resistant tuberculosis (DR-TB) (1). Several challenges, including those described by Moyo and colleagues, contribute to current abysmal outcomes, such as the treatment success rate of only $36 \%$ in this retrospective cohort. Understanding and addressing these challenges in both TB research and programming can ensure earlier detection, higher retention in care and better cure rates for adolescents with DR-TB, both with and without HIV infection.

Community-based treatment support and care programs have been implemented to address issues of adherence to treatment and retention in care for adolescents living with HIV. In Zimbabwe, Africaid's Zvandiri program, led by HIV-positive children and adolescents, works to ensure a robust continuum of care between clinic and community for adolescents and children living with HIV. The Zvandiri program operates in close collaboration with the ministry of health and child care and government and private clinics. Community adolescent treatment supporters (CATS) receive training to provide community based treatment adherence support and counseling for HIV-positive peers. Peer-led interventions provide continuous support to affected adolescents, which can help improve treatment adherence and reduce stigma associated with HIV and TB. Successful models of HIV treatment support should be adopted for adolescents undergoing treatment for DR-TB, which can last two years and result in significant side effects, some of which last beyond the end of treatment.

Several factors believed to affect adolescent treatment success rates, including stigma and major life changes such as moving, finding work and developing significant relationships and long-term commitments, might be mitigated with the development of treatment regimens that are shorter, more effective and have fewer side effects. Two new drugs, bedaquiline and delamanid have been finally approved to treat DR-TB and several phase III DR-TB treatment-shortening studies are planned or already under way. Inclusion of adolescents in phase III treatment-shortening studies should be encouraged as there is no physiological basis for their exclusion. Adolescents achieve similar levels of drug exposures as adults, present with similar forms of TB disease and tolerate adult formulations. It is imperative that sponsors of new drugs and trials testing new combinations include adolescents in their studies. Both end TB (which plans to test five all-oral, nine-month regimens containing either bedaquiline or delamanid in people with multidrug-resistant TB) and NIX-TB (which is evaluating a six-to-ninemonth regimen of bedaquiline, new drug candidate pretomanid and linezolid in people with extensively drug-resistant TB) include adolescents down to 15 and 14 years old, respectively (2-4).

Inclusion of adolescents in late-stage trials would help to expedite access to new, shorter and improved regimens for the large population of adolescents at increased risk of treatment failure and poor treatment outcomes under existing DR-TB therapies.

\section{Authors' Contributions}

Lindsay McKenna drafted this letter and submitted it. Loyce Maturu contributed content for this letter and reviewed and edited it. Erica Lessem reviewed the letter and edited it.

\section{References}

1. Moyo S, Furin JJ, Hughes J, Daniels J, Snyman L, Muller O, et al. Outcomes in Adolescents Undergoing Treatment for Drug-Re- 
sistant Tuberculosis in Cape Town, South Africa, 2008-2013. Arch Pediatr. 2014;3(3):e17934.

2. Snow KSBL. . The epidemiology of TB, TB/HIV and MDR-TB in adolescents: what is the extent of the problem? 45th Union World Conference on Lung Health. Barcelona. 2014.

3. Nachman S, Ahmed A, Amanullah F, Becerra MC, Botgros R,
Brigden G, et al. Towards early inclusion of children in tuberculosis drugs trials: a consensus statement. Lancet Infect Dis. 2015;15(6):711-20.

4. McKenna L. i-base/Treatment Action Group. 2015 pipeline report. New York: Treatment Action Group; 2015. Momentum moves the pediatric TB treatment pipeline. 